\title{
GROWTH AND INTEGRABILITY OF FOURIER TRANSFORMS ON EUCLIDEAN SPACE
}

\author{
WILLIAM O. BRAY
}

\begin{abstract}
Abstract. A fundamental theme in classical Fourier analysis relates smoothness properties of functions to the growth and/or integrability of their Fourier transform. By using a suitable class of $L^{p}$ - multipliers, a rather general inequality controlling the size of Fourier transforms for large and small argument is proved. As consequences, quantitative Riemann-Lebesgue estimates are obtained and an integrability result for the Fourier transform is developed extending ideas used by Titchmarsh in the one dimensional setting.
\end{abstract}

\section{INTRODUCTION}

A classical theme in Fourier analysis relates smoothness of functions to the growth and/or integrability of their Fourier components. In this vein, the following inequality was proved in [2]. Here the one dimensional Fourier transform is defined for integrable functions as

$$
\widehat{f}(\lambda)=\int_{\mathbb{R}} f(x) e^{-i \lambda x} d x,
$$

and extended to $L^{p}(\mathbb{R})$ in the usual fashion;

$$
\Omega_{p}[f](t)=\sup _{0<h<t}\|f(\cdot+h)+f(\cdot-h)-2 f(\cdot)\|_{p}
$$

is an $L^{p}$-modulus of continuity based on symmetric differences; and $p^{\prime}$ denotes the Hōlder conjugate index $\left(\frac{1}{p}+\frac{1}{p^{\prime}}=1\right)$.

Theorem 1.1. Let $1 \leq p \leq 2$. Then there is a constant $c_{p}>0$ such that for all $f \in L^{p}(\mathbb{R})$,

- when $p=1$,

$$
\sup _{\lambda}\left[\min \left\{1,(\lambda t)^{2}\right\}|\widehat{f}(\lambda)|\right] \leq c_{1} \Omega_{1}[f](t)
$$

- when $1<p \leq 2$,

$$
\left[\int_{\mathbb{R}} \min \left\{1,(\lambda t)^{2 p^{\prime}}\right\}|\widehat{f}(\lambda)|^{p^{\prime}} d \lambda\right]^{1 / p^{\prime}} \leq c_{p} \Omega_{p}[f](t) .
$$

The significance of this inequality stems from the presence of the minimum function that gives control over the Fourier transform for small and large $\lambda$. Indeed, for $1<p \leq 2$, the inequality may be rewritten

$$
\underbrace{\int_{|\lambda| \geq 1 / t}|\widehat{f}(\lambda)|^{p^{\prime}} d \lambda}_{\text {large } \lambda}+\underbrace{t^{2 p^{\prime}} \int_{|\lambda|<1 / t} \lambda^{2 p^{\prime}}|\widehat{f}(\lambda)|^{p^{\prime}} d \lambda}_{\text {small } \lambda} \leq c_{p}^{p^{\prime}} \Omega_{p}^{p^{\prime}}[f](t) .
$$

2000 Mathematics Subject Classification. 42B10, 42B15.

Key words and phrases. Fourier transform, multiplier, modulus of continuity, Hausdorff-Young inequality, Pick's inequality. 
As shown in [2], the estimate for large $\lambda$ yields a qualitative Riemann-Lebesgue lemma. On the other hand, from the estimate for small $\lambda$, the following integrability theorem can be proved.

Proposition 1.2. Let $1 \leq p \leq 2$ and $f \in L^{p}(\mathbb{R})$. If for some $0<\alpha \leq 2$

$$
\|f(\cdot+t)+f(\cdot-t)-2 f(\cdot)\|_{p}=O\left(t^{\alpha}\right)
$$

then $\widehat{f} \in L^{\beta}(\mathbb{R})$ provided

$$
\frac{p}{p+\alpha p-1}<\beta \leq p^{\prime}
$$

In particular, if (1.1) holds for $\alpha>1 / p$, then $\widehat{f} \in L^{1}(\mathbb{R})$ and Fourier inversion holds a.e.1

The proof of this follows from techniques used later for its generalization to higher dimensions. This result is an extension of one given in Titchmarsh [15, Theorem 84] where first order differences were used instead of the second order difference in (1.1) and consequently the restrictions are $0<\alpha \leq 1$ and $1<p \leq 2$.

Theorem 1.1 has an extension to higher dimensions [2, 3] stated below; the modulus of continuity now is based on the the spherical mean operator defined by

$$
M^{t} f(x)=\frac{1}{\omega_{n-1}} \int_{S^{n-1}} f(x+t \omega) d \omega
$$

where $S^{n-1}$ is the unit sphere in $\mathbb{R}^{n}, \omega_{n-1}$ is its measure, and $d \omega$ is induced Lebesgue measure.

Theorem 1.3. Let $n \geq 2$, let $1 \leq p \leq 2$. Then there is a constant $c_{p}>0$ such that for all $f \in L^{p}\left(\mathbb{R}^{n}\right)$,

- when $p=1$,

$$
\sup _{\xi}\left[\min \left\{1,(t|\xi|)^{2}\right\}|\widehat{f}(\xi)|\right] \leq c_{1}\left\|M^{t} f(\cdot)-f(\cdot)\right\|_{1}
$$

- when $1<p<2$,

$$
\left[\int_{\mathbb{R}^{n}} \min \left\{1,(t|\xi|)^{2 p^{\prime}}\right\}|\widehat{f}(\xi)|^{p^{\prime}} d \xi\right]^{1 / p^{\prime}} \leq c_{p}\left\|M^{t} f(\cdot)-f(\cdot)\right\|_{p}
$$

- when $p=2$,

$$
\left[\int_{\mathbb{R}^{n}} \min \left\{1,(t|\xi|)^{4}\right\}|\widehat{f}(\xi)|^{2} d \xi\right]^{1 / 2} \asymp\left\|M^{t} f(\cdot)-f(\cdot)\right\|_{2} .
$$

(Here $\asymp$ means the left hand side is bounded above and below by a positive constant times the right hand side.)

Aside from the use of the Hausdorff-Young inequality/Plancherel theorem, the proof of Theorem 1.3 depends on two other key ideas:

- The multiplier identity

$$
\widehat{M^{t} f}(\xi)=\widehat{f}(\xi) j_{\nu}(t|\xi|),
$$

where $j_{\nu}$ is the spherical Bessel function of order $\nu=\frac{n-2}{2}$,

$$
j_{\nu}(r)=2^{\nu} \Gamma(\nu+1) r^{-\nu} J_{\nu}(r)
$$

${ }_{1}$ Fourier inversion actually holds everywhere. The Fourier inversion integral defines a continuous function which is equal to $f$ a.e. This remark also applies to Corollaries 3.5 4.4 and 4.6 
$J_{\nu}$ being Bessel function of the first kind.

- The estimate (for $\alpha>-1 / 2$ )

$$
1-j_{\alpha}(\lambda) \asymp \min \left\{1, \lambda^{2}\right\},
$$

derived from the Mehler representation of Bessel functions (see [3]).

The use of spherical means in Theorem 1.3 was motivated by the work of Gioev [8]. Defining a modulus of continuity using the spherical mean operator seems to have first been used in the work of Platonov [13] where a generalization of a different result of Titchmarsh was proved. Theorem 1.3 (and a generalization) was also obtained by Ditzian [5] using related methods and from an approximation theory perspective. From that work, it is clear that the term $\left\|M^{t} f(\cdot)-f(\cdot)\right\|_{p}$ measures second order smoothness and can be thought of as an analog for $L^{p}\left(\mathbb{R}^{n}\right), n \geq 2$, of the second order difference operator used in Theorem 1.1. In later work, Ditzian [6] obtained a variation of Theorem 1.3 replacing the HausdorffYoung inequality by the Hardy-Littlewood inequality. Another variation was obtained by Gorbachev and Tikhonov [9] making use of Pick's inequality. Further comments regarding these works and the relation to the results of the present paper will be given later.

The focus of the current paper is on generalizations of Theorem 1.3 and on extensions of Proposition 1.2 to higher dimensions. Section 2 gives a wide class of multiplier operators for which an analog of Theorem 1.3 is valid as well as variations in the vein of [6, 9]. In section 3 , the analog of Proposition 1.2 is developed. Finally, a couple of variations are presented in section 4. Many of the results developed here have analogs in rank one symmetric spaces and more generally, Damek-Ricci spaces; details will appear in a sequel to this paper.

\section{Generalizations \& Variations of Theorem 1.3}

Let $\mu$ be a finite Borel measure on $\mathbb{R}^{n}, n \geq 2$, we assume $\int_{\mathbb{R}^{n}} d \mu=1$. The total variation of $\mu$ is denoted $\|\mu\|_{\mathcal{M}}=\int_{\mathbb{R}^{n}} d|\mu|$. Using convolution, $\mu$ defines a Fourier multiplier operator on $L^{1}\left(\mathbb{R}^{n}\right)$; on the Fourier transform side, the multiplier is given by the Fourier transform of the measure $\widehat{\mu}(\xi)$. For $t>0$, the dilation of $\mu$ is defined via the following natural formula:

$$
f \in L^{1}\left(\mathbb{R}^{n}\right), \int_{\mathbb{R}^{n}} f(x) d \mu_{t}(x)=\int_{\mathbb{R}^{n}} f(t x) d \mu(x) .
$$

The measure $\mu_{t}$ defines a Fourier multiplier operator on $L^{1}\left(\mathbb{R}^{n}\right)$ through convolution:

$$
M_{\mu}^{t} f(x)=\left(f * \mu_{t}\right)(x)=\int_{\mathbb{R}^{n}} f(x-t y) d \mu(y) .
$$

It is immediate that $\left\|M_{\mu}^{t} f\right\|_{1} \leq\|f\|_{1}\|\mu\|_{\mathcal{M}}$. From standard theory (e.g. [10]) the operator is also a Fourier multiplier on $L^{p}\left(\mathbb{R}^{n}\right)$ for all $1 \leq p \leq \infty$; the corresponding multiplier is given by $\widehat{\mu_{t}}(\xi)=\widehat{\mu}(t \xi)$. The following result is an extension of classical approximate identity ideas.

Proposition 2.1. Let $\mu$ be as above and $1 \leq p<\infty$. Then for all $f \in L^{p}\left(\mathbb{R}^{n}\right)$, $\| M_{\mu}^{t} f-$ $f \|_{p} \rightarrow 0$ as $t \rightarrow 0$.

Proof. Consider the case $p>1 ; p=1$ is similar. Applying Hōlder's inequality,

$$
\left|M_{\mu}^{t} f(x)-f(x)\right| \leq\|\mu\|_{\mathcal{M}}^{1 / p^{\prime}}\left(\int_{\mathbb{R}^{n}}|f(x-t y)-f(x)|^{p} d|\mu|(y)\right)^{1 / p} .
$$


Raising both sides to the power $p$, integrating over $\mathbb{R}^{n}$, and interchanging orders of integration yields:

$$
\left\|M_{\mu}^{t} f-f\right\|_{p}^{p} \leq \|\left.\mu\right|_{\mathcal{M}} ^{p-1} \int_{\mathbb{R}^{n}}\left[\int_{\mathbb{R}^{n}}|f(x-t y)-f(x)|^{p} d x\right] d|\mu|(y) .
$$

Let $\omega_{p}[f](h)=\|f(\cdot+h)-f(\cdot)\|_{p}$ be the $L^{p}$-modulus of continuity; it is known that $\omega_{p}[f](h) \leq 2\|f\|_{p}$ and $\omega_{p}[f](h) \rightarrow 0$ as $|h| \rightarrow 0$. Let $R>0$ and split the outer integral in (2.1) into two integrals, $I_{1}(t, R)$ over $|y|<R$ and $I_{2}(t, R)$ over $|y| \geq R$, respectively. Let $\varepsilon>0$. We have

$$
I_{2}(t, R) \leq\left(2\|f\|_{p}\right)^{p}\|\mu\|_{\mathcal{M}}^{p-1} \int_{|y| \geq R} d|\mu|(y)
$$

hence there is an $R>0$ such that $I_{2}(t, R)<(\varepsilon / 2)^{p}$. Choose $\delta>0$ such that if $|h|<\delta$, then $\omega_{p}[f](h)<\varepsilon /\left(2\|\mu\|_{\mathcal{M}}^{p}\right)$. Since $R$ has been fixed, choose $t_{0}>0$ such that $t_{0} R<\delta$. Then for $t<t_{0}$

$$
I_{2}(t, R)=\|\mu\|_{\mathcal{M}}^{p-1} \int_{|y|<R} \omega_{p}[f](t y)^{p} d|\mu|(y)<\left(\frac{\varepsilon}{2}\right)^{p} .
$$

Putting the estimates together completes the proof.

Setting the stage for putting Theorem 1.3 from the introduction in general form, let $\mu$ be a finite Borel measure on $\mathbb{R}^{n}$ with $\int_{\mathbb{R}^{n}} d \mu=1$ and whose Fourier multiplier satisfies the estimate

$$
|1-\widehat{\mu}(\xi)| \asymp \min \left\{1,|\xi|^{2 \sigma}\right\},
$$

for some $\sigma>0$. The class of such measures is denoted $\mathcal{K}_{\sigma}=\mathcal{K}_{\sigma}\left(\mathbb{R}^{n}\right)$.

Theorem 2.2. Let $1 \leq p \leq 2$ and let $\mu \in \mathcal{K}_{\sigma}$. Then there is a constant $c_{p}>0$ such that for all $f \in L^{p}\left(\mathbb{R}^{n}\right)$,

(1) when $p=1$,

$$
\sup _{\xi \in \mathbb{R}^{n}}\left[\min \left\{1,(t|\xi|)^{2 \sigma}\right\}|\widehat{f}(\xi)|\right] \leq c_{1}\left\|M_{\mu}^{t} f-f\right\|_{1}
$$

(2) when $1<p<2$,

$$
\left[\int_{\mathbb{R}^{n}} \min \left\{1,(t|\xi|)^{2 \sigma p^{\prime}}\right\}|\widehat{f}(\xi)|^{p^{\prime}} d \xi\right]^{1 / p^{\prime}} \leq c_{p}\left\|M_{\mu}^{t} f-f\right\|_{p}
$$

(3) when $p=2$,

$$
\left[\int_{\mathbb{R}^{n}} \min \left\{1,(t|\xi|)^{4 \sigma}\right\}|\widehat{f}(\xi)|^{2} d \xi\right]^{1 / 2} \asymp\left\|M_{\mu}^{t} f-f\right\|_{2} .
$$

Proof. We give the proof of item 2. Since $\widehat{M_{\mu}^{t} f}(\xi)=\widehat{f}(\xi) \widehat{\mu}(t \xi)$ we have

$$
\left(\widehat{M_{\mu}^{t} f-} f\right)(\xi)=[\widehat{\mu}(t \xi)-1] \widehat{f}(\xi) .
$$

Applying the Hausdorff-Young inequality we obtain

$$
\left[\int_{\mathbb{R}^{n}}|1-\widehat{\mu}(t \xi)|^{p^{\prime}}|\widehat{f}(\xi)|^{p^{\prime}} d \xi\right]^{1 / p^{\prime}} \leq c_{p}\left\|M_{\mu}^{t} f-f\right\|_{p} .
$$

The inequality follows by from the estimate (2.2). Item 1 follows in a similar fashion using the $L^{1}$-estimate $\|\widehat{f}\|_{\infty} \leq\|f\|_{1}$, and item 3 follows using the Plancherel theorem. 
Below are several examples that provide concrete realization of this result.

Example 2.3. Theorem 1.3 from the introduction is a corollary of the above theorem as follows. Let $d \mu=d \omega / \omega_{n-1}$, where $d \omega$ is the usual surface measure on the unit sphere in $\mathbb{R}^{n}$. Then $M_{\mu}^{t}=M^{t}$, the spherical mean operator. In this case $\widehat{\mu}(\xi)=j_{\nu}(|\xi|)$ where $\nu=\frac{n-2}{2}$, and we have the estimate $1-j_{\nu}(r) \asymp \min \left\{1, r^{2}\right\}$, so $\mu \in \mathcal{K}_{1}$. For later reference, the following identity was used in [2] to derive this estimate: for $\alpha>-1 / 2$,

$$
1-j_{\alpha}(\lambda)=\frac{4 \Gamma(\alpha+1)}{\sqrt{\pi} \Gamma(\alpha+1 / 2)} \int_{0}^{1}\left(1-s^{2}\right)^{\alpha-1 / 2} \sin ^{2} \frac{\lambda s}{2} d s .
$$

This example can be extended by iteration as follows. If $\mu^{(l)}$ denotes the convolution of $\mu$ with itself $l$-times, the corresponding multiplier is $\left(j_{\nu}(|\xi|)\right)^{l}$ and satisfies the same estimate as above for $\widehat{\mu}(\xi)$. The corresponding operator is the $l^{\text {th }}$-iterate of the spherical mean operator, denoted $M_{l}^{t}$.

Example 2.4. Let the Lebesgue measure of a set $A$ be denoted $|A|$, and let $\Omega_{n}$ be the measure of the unit ball $B(0,1)$. For a measurable set $A \subset \mathbb{R}^{n}$, define $\mu(A)=\mid A \cap$ $B(0,1) \mid / \Omega_{n}$. To this measure, corresponds the averaging operator

$$
\mathcal{A}^{t} f(x)=\int_{\mathbb{R}^{n}} f(x-t y) d \mu(y)=\frac{1}{\Omega_{n}} \int_{B(0,1)} f(x-t y) d y .
$$

An easy computation shows that $\widehat{\mu}(\xi)=j_{\nu+1}(|\xi|)$ where $\nu=\frac{n-2}{2}$ and hence, $\mu \in \mathcal{K}_{1}$ from previous considerations.

Example 2.5. Let $C$ be the surface of the cube in $\mathbb{R}^{n}$ centered at the origin whose $2 n$ faces each have area one. Here we take $d \mu=d S /(2 n)$, where $d S$ is the surface measure on the boundary $\partial C$ of $C$ induced by Lebesgue measure. A simple, albeit tedious, calculation gives the multiplier:

$$
\widehat{\mu}(\xi)=\frac{1}{2 n} \int_{\partial C} e^{-i(\xi \cdot x)} d S(x)=\frac{1}{2^{n-1} n} \sum_{k=1}^{n} \cos \frac{\xi_{k}}{2} \prod_{l=1, l \neq k}^{n} \frac{\sin \frac{\xi_{l}}{2}}{\xi_{l}} .
$$

In this case we have $|1-\widehat{\mu}(\xi)| \asymp \min \left\{1,|\xi|^{2}\right\}$ and $\mu \in \mathcal{K}_{1}$.

The above examples are unified and generalized as follows. Let $K \subset \mathbb{R}^{n}$ be a compact connected symmetric $(-x \in K$ if $x \in K)$ set with non-empty interior and whose boundary $S$ is a piecewise smooth regular surface. The latter means that $S=\cup_{l=1}^{m} S_{l}$, where each $S_{l}$ is a smooth surface that is given as the level surface of a smooth function $F_{l}(x)=0$ whose gradient never vanishes on $S_{l}$.

Proposition 2.6. Let $K \subset \mathbb{R}^{n}$ be a compact set as above.

(1) Let $\mu$ be the normalized surface measure on $S$ induced by Lebesgue measure. Then $\mu \in \mathcal{K}_{1}$.

(2) Let $\mu$ be the measure on $\mathbb{R}^{n}$ defined by $\mu(A)=|A \cap K| /|K|$, for any measurable set $A \subset \mathbb{R}^{n}$. Then $\mu \in \mathcal{K}_{1}$.

Proof. We prove (1); the proof of (2) is similar. Denote normalized surface measure on $S$ by $d S$. Since $K$ is a symmetric set,

$$
\widehat{\mu}(\xi)=\int_{S} \cos \xi \cdot x d S(x)
$$


and consequently

$$
1-\widehat{\mu}(\xi)=2 \int_{S} \sin ^{2} \frac{\xi \cdot x}{2} d S(x) .
$$

Clearly $|1-\widehat{\mu}(\xi)| \leq 2$. We claim that $1-\widehat{\mu}(\xi)=0$ only at the origin and consequently, given $r>0$, there is a constant $c>0$ (dependent on $r$ ) such that $|1-\widehat{\mu}(\xi)| \geq c$ provided $|\xi|>r$. To prove the claim in the case where $S$ is a smooth surface, suppose $\xi \neq 0$ such that $1-\widehat{\mu}(\xi)=0$. Since $x \rightarrow \frac{\xi \cdot x}{2}$ is continuous on $S, \sin \frac{\xi \cdot x}{2}=0$ for all $x \in S$. However, this implies that $S$ if contained in some plane $\xi \cdot x=2 \pi k$, where $k$ is an integer, a contradiction. In the case where $S=\cup_{m} S_{m}$, where each $S_{m}$ is smooth, the above ideas imply that the entire surface is contained in a union of parallel planes, again a contradiction. To obtain the rest of the estimate, take $R>0$ such that $K \subset\{x:|x|<R\}$ and suppose $|\xi|<\pi / R$. Then from the inequality $\frac{2}{\pi}|u| \leq|\sin u| \leq u$ for $|u| \leq \pi / 2$, it follows that for all $x \in S$,

$$
\frac{1}{\pi^{2}}(\xi \cdot x)^{2} \leq \sin ^{2} \frac{\xi \cdot x}{2} \leq \frac{1}{4}(\xi \cdot x)^{2} .
$$

Integrating over $S$, there are positive constants $c$ and $c^{\prime}$ such that

$$
c N^{2}(\xi) \leq|1-\widehat{\mu}(\xi)| \leq c^{\prime} N^{2}(\xi),
$$

where $N(\xi)=\left[\int_{S}(\xi \cdot x)^{2} d S(x)\right]^{1 / 2}$. It is easy to see that $\xi \rightarrow N(\xi)$ is a norm on $\mathbb{R}^{n}$ and since all norms on $\mathbb{R}^{n}$ are equivalent, there are positive constants $d$ and $d^{\prime}$ such that $d|\xi| \leq N(\xi) \leq d^{\prime}|\xi|$. Thus, for $|\xi|<\pi / R$, there are constants $c_{1}$ and $c_{2}$ such that

$$
c_{1}|\xi|^{2} \leq|1-\widehat{\mu}(\xi)| \leq c_{2}|\xi|^{2}
$$

and the result follows.

The proposition and its proof have easy generalization by multiplying the measures in (1) or (2) by suitable functions. For example, the result given in (1) is valid for measures of the form $d \mu=\varphi d S$, where $\phi$ is a non-negative continuous function on $\mathbb{R}^{n}$ with $\int_{S} \varphi d S=1$.

Other examples of measures in some $\mathcal{K}_{\sigma}$ come from classical approximate identities. The following example is illustrative.

Example 2.7. Let $d \mu=H(x) d x$, where

$$
H(x)=\frac{1}{2^{n} \pi^{n / 2}} e^{-|x|^{2} / 4}
$$

(notice that $\int_{\mathbb{R}^{n}} H(x) d x=1$ ). In this case the multiplier is $\widehat{H}(\xi)=e^{-|\xi|^{2}}$ and the estimate $1-e^{-|\xi|^{2}} \asymp \min \left\{1,|\xi|^{2}\right\}$ is elementary. The operator of interest is given by: $M_{\mu}^{t} f(x)=$ $\left(f * H_{t}\right)(x)$. Comparing the conclusion of Theorem 2.2 in the $L^{2}$ case for the Examples 2.3 and 2.7 above leads to the following interesting conclusion: for $f \in L^{2}\left(\mathbb{R}^{n}\right)$,

$$
\left\|f * H_{t}-f\right\|_{2} \asymp\left\|M^{t} f-f\right\|_{2} .
$$

In other words, the approximations $f * H_{t}$ and $M^{t} f$ have the same rate of approximation in $L^{2}$-norm.

Notice that if we dilate using $t^{1 / 2}$ instead of $t$, then $H_{t^{1 / 2}}(x)$ is the heat kernel, $H_{t^{1 / 2}} * f$ is the solution of the heat equation $u_{t}=\Delta u$ ( $\Delta$ being the Laplacian) with initial data $u(x, 0)=f(x)$, and further, the estimates in the theorem must be modified accordingly.

For the results of section 3, measures are needed which lead to higher values of $\sigma$ than in the previous examples. The following two results effectively achieve this. The first is based on the iterate $\left(I-M_{\mu}^{t}\right)^{l}$ for appropriate measures $\mu$. 
Proposition 2.8. Let $\mu$ be as in Proposition 2.6 and $l \geq 1$. Define

$$
\mu^{\prime}=\sum_{k=1}^{l}(-1)^{k+1}\left(\begin{array}{c}
l \\
l-k
\end{array}\right) \mu^{(k)},
$$

where $\mu^{(k)}$ is convolution of $\mu$ with itself $k$-times. Then $\mu^{\prime} \in \mathcal{K}_{l}$ and moreover, the corresponding operator is given by

$$
M_{\mu^{\prime}}^{t} f(x)=\sum_{k=1}^{l}(-1)^{k}\left(\begin{array}{c}
l \\
l-k
\end{array}\right) M_{\mu^{(k)}}^{t} f(x) .
$$

Proof. From the binomial theorem,

$$
\begin{aligned}
1-\widehat{\mu^{\prime}}(\xi) & =1-\sum_{k=1}^{l}(-1)^{k+1}\left(\begin{array}{c}
l \\
l-k
\end{array}\right) \widehat{\mu}^{k}(\xi) \\
& =(1-\widehat{\mu}(\xi))^{l} .
\end{aligned}
$$

The result now follows as $\mu \in \mathcal{K}_{1}$.

An alternative approach to constructing measures in $\mathcal{K}_{\sigma}$ with $\sigma>1$ generalizes operators used in [4] for problems in approximation theory. These operators are described as follows.

Example 2.9. The identity (2.3) was originally used in obtaining the estimate $1-j_{\alpha}(\lambda) \asymp$ $\min \left\{1, \lambda^{2}\right\}$. In particular, the presence of the squared sine term on the right hand side is key. The idea here is to develop an operator where this term is replaced by sine to a higher power and leads to and motivates operators introduced by Dai and Ditzian [4. Let $l \geq 1$ be an integer. Then using Euler's identity, the binomial theorem, and standard manipulation yields the trigonometric identity [4]

$$
\begin{aligned}
4^{l}\left(\begin{array}{c}
2 l \\
l
\end{array}\right)^{-1} \sin ^{2 l} \frac{y}{2} & =1-2\left(\begin{array}{c}
2 l \\
l
\end{array}\right)^{-1} \sum_{k=1}^{l}(-1)^{k+1}\left(\begin{array}{c}
2 l \\
l-k
\end{array}\right) \cos k y \\
& =1-v_{l}(y) .
\end{aligned}
$$

For $\alpha>-1 / 2$, define

$$
\begin{aligned}
j_{\alpha, l}(\lambda) & =\frac{2 \Gamma(\alpha+1)}{\sqrt{\pi} \Gamma(\alpha+1 / 2)} \int_{0}^{1}\left(1-y^{2}\right)^{\alpha-1 / 2} v_{l}(\lambda y) d y \\
& =2\left(\begin{array}{c}
2 l \\
l
\end{array}\right)^{-1} \sum_{k=1}^{l}(-1)^{k+1}\left(\begin{array}{c}
2 l \\
l-k
\end{array}\right) j_{\alpha}(k \lambda) .
\end{aligned}
$$

With $\alpha=\nu=\frac{n-2}{2}$, this is the Fourier multiplier corresponding to the measure

$$
\mu=2\left(\begin{array}{c}
2 l \\
l
\end{array}\right)^{-1} \sum_{k=1}^{l}(-1)^{k+1}\left(\begin{array}{c}
2 l \\
l-k
\end{array}\right) \omega_{k},
$$

where $\omega$ is normalized surface measure on the unit sphere and $\omega_{k}$ is its dilation by $k$. The corresponding operator is given by

$$
V_{l}^{t} f(x)=2\left(\begin{array}{c}
2 l \\
l
\end{array}\right)^{-1} \sum_{k=1}^{l}(-1)^{k+1}\left(\begin{array}{c}
2 l \\
l-k
\end{array}\right) M^{k t} f(x) .
$$


This operator is precisely the one introduced in [4] for problems in approximation theory and used in [5, 6, 9] in obtaining generalization and variations of Theorem 1.3. In this case the multiplier estimate is $|1-\widehat{\mu}(\xi)| \asymp \min \left\{1,|\xi|^{2 l}\right\}$ as follows from the integral defining $j_{\nu, l}$ and the above trigonometric identity.

Proposition 2.10. Let $\mu$ be as in Proposition 2.6 and $l \geq 1$. Define

$$
\mu^{\prime}=2\left(\begin{array}{c}
2 l \\
l
\end{array}\right)^{-1} \sum_{k=1}^{l}(-1)^{k+1}\left(\begin{array}{c}
2 l \\
l-k
\end{array}\right) \mu_{k},
$$

where $\mu_{k}$ is dilation of $\mu$ by $k$. Then $\mu^{\prime} \in \mathcal{K}_{l}$ and the corresponding operator is given by

$$
M_{\mu^{\prime}}^{t} f(x)=2\left(\begin{array}{c}
2 l \\
l
\end{array}\right)^{-1} \sum_{k=1}^{l}(-1)^{k+1}\left(\begin{array}{c}
2 l \\
l-k
\end{array}\right) M_{\mu}^{k t} f(x) .
$$

Proof. We have

$$
\begin{aligned}
\widehat{\mu^{\prime}}(\xi) & =2\left(\begin{array}{c}
2 l \\
l
\end{array}\right)^{-1} \sum_{k=1}^{l}(-1)^{k+1}\left(\begin{array}{c}
2 l \\
l-k
\end{array}\right) \widehat{\mu}(k \xi) \\
& =\int_{\mathbb{R}^{n}}\left[2\left(\begin{array}{c}
2 l \\
l
\end{array}\right)^{-1} \sum_{k=1}^{l}(-1)^{k+1}\left(\begin{array}{c}
2 l \\
l-k
\end{array}\right) \cos k(x \cdot \xi)\right] d \mu(x)
\end{aligned}
$$

and hence

$$
1-\widehat{\mu^{\prime}}(\xi)=4^{l}\left(\begin{array}{c}
2 l \\
l
\end{array}\right)^{-1} \int_{\mathbb{R}^{n}} \sin ^{2 l} \frac{x \cdot \xi}{2} d \mu(x) .
$$

The result follows by applying ideas used in the proof of Proposition 2.6.

Remark 2.11. The right hand side of the estimates given in Theorem 2.2 take the form $\left\|\left(I-M_{\mu}^{t}\right)^{l} f\right\|_{p}$ and $\left\|M_{\mu^{\prime}}^{t} f-f\right\|_{p}$ in the case of the measures given by Proposition 2.8 and Proposition 2.10, respectively. The advantage of the latter lies in the fact that the operator $M_{\mu^{\prime}}^{t}$ is a linear combination of dilates of a single operator.

This section concludes with the following result generalizing Theorem 2.2 and results given in [6, 9] for the operators $V_{l}^{t}$ of Example 2.9.

Theorem 2.12. Let $\mu \in \mathcal{K}_{\sigma}$.

(1) Let $1<p \leq 2, p \leq q \leq p^{\prime}$, and $f \in L^{p}\left(\mathbb{R}^{n}\right)$. Then for all $f \in L^{p}\left(\mathbb{R}^{n}\right)$, $|\xi|^{n\left(1-\frac{1}{p}-\frac{1}{q}\right)} \widehat{f}(\xi) \in L^{q}\left(\mathbb{R}^{n}\right)$ and there is a constant $c_{p, q}>0$ such that

$$
\left[\int_{\mathbb{R}^{n}} \min \left\{1,(t|\xi|)^{2 \sigma q}\right\}|\xi|^{q n\left(1-\frac{1}{p}-\frac{1}{q}\right)}|\widehat{f}(\xi)|^{q} d \xi\right]^{1 / q} \leq c_{p, q}\left\|M_{\mu}^{t} f-f\right\|_{p} .
$$

(2) Let $2 \leq p<\infty$ and let $q>1$ with $\max \left\{q, q^{\prime}\right\} \leq p$. If $f \in L^{p}\left(\mathbb{R}^{n}\right)$ with $|\xi|^{n\left(1-\frac{1}{p}-\frac{1}{q}\right)} \widehat{f}(\xi) \in$ $L^{q}\left(\mathbb{R}^{n}\right)$, then there is a constant $c_{p, q}>0$ such that

$$
\left\|M_{\mu}^{t} f-f\right\|_{p} \leq c_{p, q}\left[\int_{\mathbb{R}^{n}} \min \left\{1,(t|\xi|)^{2 \sigma q}\right\}|\xi|^{q n\left(1-\frac{1}{p}-\frac{1}{q}\right)}|\widehat{f}(\xi)|^{q} d \xi\right]^{1 / q} .
$$


The proof uses the following special cases of Pick's inequality [1, 4] instead of the Hausdorff-Young theorem. Under the assumptions of item (1) above,

$$
\left[\int_{\mathbb{R}^{n}}|\xi|^{q n\left(1-\frac{1}{p}-\frac{1}{q}\right)}|\widehat{f}(\xi)|^{q} d \xi\right]^{1 / q} \leq c_{p, q}\|f\|_{p} .
$$

In the case $q=p^{\prime}$, this is just the Hausdorff-Young inequality; the case $q=p$, it is the HardyLittlewood inequality. Likewise, under the assumptions of item (2), Pick's inequality takes the form

$$
\|f\|_{p} \leq c_{p, q}\left[\int_{\mathbb{R}^{n}}|\xi|^{q n\left(1-\frac{1}{p}-\frac{1}{q}\right)}|\widehat{f}(\xi)|^{q} d \xi\right]^{1 / q} .
$$

In the case where $q=p^{\prime}$, this is the dual form of the Hausdorff-Young inequality. To obtain the conclusions of the theorem, we apply the above inequalities to $M_{\mu}^{t} f-f$ and proceed as in Theorem 2.2.

Remark 2.13. For the operator $V_{l}^{t}$ of Example 2.9, the above result was proved in [6] in the case $q=p$; the cases $p \leq q \leq p^{\prime}$ were considered in [9] for the same operators. In both cases the proof was based on the connection between the operator and $K$-functionals associated with the Laplacian. Specifically, in [4] it was shown that

$$
\left\|V_{l}^{t} f-f\right\|_{p} \asymp K_{l}\left(f, \Delta^{l}, t^{2 l}\right)_{p},
$$

where $\Delta$ is the Laplacian on $\mathbb{R}^{n}$ and

$$
K_{l}\left(f, \Delta^{l}, t^{2 l}\right)_{p}=\inf \left\{\|f-g\|_{p}+t^{2 l}\left\|\Delta^{l} g\right\|_{p}\right\},
$$

the infinum taken over all $g \in L^{p}\left(\mathbb{R}^{n}\right)$ such that $\Delta^{l} g \in L^{p}\left(\mathbb{R}^{n}\right)$. As such, the $K$-functional gives a gauge on the order of smoothness of approximations and the equivalence (2.5) gives the same interpretation to the differences $\left\|V_{l}^{t} f-f\right\|_{p}$. The proofs given in [5, 6, 9] of our results in the case of the operators $V_{l}^{t}$ depend on (2.5) as well as relationships between the $K$-functional and generalized Bochner-Riesz means introduced in [4]. Our proofs given above are far simpler and in the vein of classical Fourier analysis. It would be interesting find $K$-functional relations for the operators of Propositions 2.8 and 2.10. (The techniques used in [4] use the specific structure of the multiplier associated with $V_{l}^{t}$ and do not shed light on this problem; see also [7].)

\section{Estimates \& Integrability of Fourier Transform}

Herein we present applications of the theorems of the previous section. Quantitative Riemann-Lebesgue estimates a deduced from the "large $\xi$ " part of the estimates given in Theorems 2.2 and 2.12 and an integrability result is deduced from the "small $\xi$ " part.

3.1. Riemann-Lebesgue Type Estimates. The following result is a general form of one given in [2] for the spherical mean operator. This result is immediate from the estimates given in Theorem 2.2 .

Corollary 3.1. Let $\mu \in \mathcal{K}_{\sigma}$ for some $\sigma>0$ and let $1 \leq p \leq 2$. Then for any $f \in L^{p}\left(\mathbb{R}^{n}\right)$ :

- when $p=1$,

$$
\sup _{|\xi|>1 / t}|\widehat{f}(\xi)| \leq c_{1}\left\|M_{\mu}^{t} f-f\right\|_{1}
$$


- when $1<p \leq 2$,

$$
\int_{|\xi|>1 / t}|\widehat{f}(\xi)|^{p^{\prime}} d \xi \leq c_{p}\left\|M_{\mu}^{t} f-f\right\|_{p}^{p^{\prime}} .
$$

The following variation is apparent from Theorem 2.12 , in original form it appeared in [6, 9].

Corollary 3.2. Let $\mu \in \mathcal{K}_{\sigma}$ for some $\sigma>0$, let $1<p \leq 2$ and $p \leq q \leq p^{\prime}$. Then for any $f \in L^{p}\left(\mathbb{R}^{n}\right)$, there is a constant $c_{p}>0$ such that

$$
\int_{|\xi|>1 / t}|\xi|^{q n\left(1-\frac{1}{p}-\frac{1}{q}\right)}|\widehat{f}(\xi)|^{q} d \xi \leq c_{p}\left\|M_{\mu}^{t} f-f\right\|_{p}^{q} .
$$

When $p=2$ the estimates in the two corollaries above are identical. A specialization is possible in this case making use of a Lipschitz condition. The following result generalizes one found in [13] and rediscovered in [8]; the result in one dimension dates back to Titchmarsh [15, Theorem 85].

Proposition 3.3. Let $\mu \in \mathcal{K}_{\sigma}$ for some $\sigma>0$ and let $0<\alpha \leq 2 \sigma$. Then for $f \in L^{2}\left(\mathbb{R}^{n}\right)$,

$$
\left\|M_{\mu}^{t} f-f\right\|_{2}=O\left(t^{\alpha}\right)(t \rightarrow 0)
$$

if and only if

$$
\int_{|\xi|>1 / t}|\widehat{f}(\xi)|^{2} d \xi=O\left(t^{2 \alpha}\right)(t \rightarrow 0) .
$$

Proof. That (3.1) implies (3.2) is immediate from the previous corollaries. For the other implication we modify the technique given in [13. Let

$$
F(\lambda)=\int_{S^{n-1}}|\widehat{f}(\lambda \omega)|^{2} d \omega
$$

where $d \omega$ is the usual surface measure on $S^{n-1}$. From the $L^{2}$-estimate in the preceding corollary,

$$
\begin{aligned}
\left\|M_{\mu}^{t} f-f\right\|_{2}^{2} & =O\left(\int_{1 / t}^{\infty} F(\lambda) \lambda^{n-1} d \lambda+t^{4 \sigma} \int_{0}^{1 / t} \lambda^{4 \sigma} F(\lambda) \lambda^{n-1} d \lambda\right) \\
& =O\left(t^{2 \alpha}\right)+O\left(t^{4 \sigma} \int_{0}^{1 / t} \lambda^{4 \sigma} F(\lambda) \lambda^{n-1} d \lambda\right) .
\end{aligned}
$$

Now set $\varphi(r)=\int_{r}^{\infty} F(\lambda) \lambda^{n-1} d \lambda$, then integration by parts and the hypothesis yield:

$$
\begin{aligned}
t^{4 \sigma} \int_{0}^{1 / t} \lambda^{4 \sigma} F(\lambda) \lambda^{n-1} d \lambda & =-t^{4 \sigma} \int_{0}^{1 / t} \lambda^{4 \sigma} \varphi^{\prime}(\lambda) d \lambda \\
& =-t^{4 \sigma}\left[t^{-4 \sigma} \varphi(1 / t)-4 \sigma \int_{0}^{1 / t} \lambda^{4 \sigma-1} \varphi(\lambda) d \lambda\right] \\
& =O\left(t^{2 \alpha}\right)+O\left(t^{4 \sigma} \int_{0}^{1 / t} \lambda^{4 \sigma-2 \alpha-1} d \lambda\right) \\
& =O\left(t^{2 \alpha}\right) .
\end{aligned}
$$

Putting the estimates together completes the proof. 
3.2. An Integrabilty Result. Let $f \in L^{p}\left(\mathbb{R}^{n}\right)$ for some $1 \leq p \leq 2$, then in spherical coordinates $\widehat{f}(\lambda \omega)$ is defined a.e. and we set

$$
F(\lambda)=\left\{\begin{array}{cc}
\max _{\omega \in S^{n-1}}|\widehat{f}(\lambda \omega)|, & p=1 \\
{\left[\int_{S^{n-1}}|\widehat{f}(\lambda \omega)|^{p^{\prime}} d \omega\right]^{1 / p^{\prime}},} & 1<p \leq 2
\end{array} .\right.
$$

Below is a general integrability theorem concerning $F(\lambda)$. The Lipschitz condition in the hypothesis has natural limitations because of the nature of the inequalities in Theorem 2.2 for small $|\xi|$, e.g., in the case $1<p \leq 2$, we have

$$
\int_{|\xi|<1 / t}|\xi|^{2 \sigma p^{\prime}}|\widehat{f}(\xi)|^{p^{\prime}} d \xi \leq c_{p}^{p^{\prime}}\left(\frac{\left\|M_{\mu}^{t} f-f\right\|_{p}}{t^{2 \sigma}}\right)^{p^{\prime}} .
$$

Proposition 3.4. Let $\mu \in K_{\sigma}$ and $f \in L^{p}\left(\mathbb{R}^{n}\right)$ for some $1 \leq p \leq 2$. If for some $0<\alpha \leq 2 \sigma$,

$$
\left\|M_{\mu}^{t} f-f\right\|_{p}=O\left(t^{\alpha}\right), t \rightarrow 0,
$$

then $F \in L^{\beta}\left((0, \infty), \lambda^{n-1} d \lambda\right)$ provided

$$
\frac{n p}{n p+\alpha p-n}<\beta \leq p^{\prime} .
$$

Proof. Set $\Lambda=t^{-1}$, and rewrite (3.3) as,

$$
\int_{1}^{\Lambda} \lambda^{2 \sigma p^{\prime}} F(\lambda)^{p^{\prime}} \lambda^{n-1} d \lambda \leq C \Lambda^{(2 \sigma-\alpha) p^{\prime}} .
$$

Take $\beta<p^{\prime}$ and let

$$
\phi(\Lambda)=\int_{1}^{\Lambda}\left[\lambda^{2 \sigma} F(\lambda)\right]^{\beta} \lambda^{n-1} d \lambda .
$$

Applying Holder's inequality we deduce ( $C$ designates a generic constant which may change from expression to expression):

$$
\begin{aligned}
\phi(\Lambda) & =\int_{1}^{\Lambda}\left[\lambda^{2 \sigma} F(\lambda)\right]^{\beta} \lambda^{(n-1) \frac{\beta}{p^{\prime}}} \lambda^{(n-1)\left(1-\frac{\beta}{p^{\prime}}\right)} d \lambda \\
& \leq C\left(\int_{1}^{\Lambda} \lambda^{2 \sigma p^{\prime}} F(\lambda)^{p^{\prime}} \lambda^{n-1} d \lambda\right)^{\beta / p^{\prime}}\left(\int_{1}^{\Lambda} \lambda^{n-1} d \lambda\right)^{1-\beta / p^{\prime}} \\
& \leq C \Lambda^{2 \sigma \beta-\alpha \beta}\left(1+\Lambda^{n}\right)^{1-\frac{\beta}{p^{\prime}}} \\
& =O\left(\Lambda^{2 \sigma \beta+n-\alpha \beta-\frac{n \beta}{p^{\prime}}}\right) .
\end{aligned}
$$

Integration by parts yields the identity,

$$
\begin{aligned}
\int_{1}^{\Lambda} F(\lambda)^{\beta} \lambda^{n-1} d \lambda & =\int_{1}^{\Lambda} \lambda^{-2 \sigma \beta} \phi^{\prime}(\lambda) d \lambda \\
& =\Lambda^{-2 \sigma \beta} \phi(\Lambda)+2 \beta \sigma \int_{1}^{\Lambda} \lambda^{-2 \sigma \beta-1} \phi(\lambda) d \lambda
\end{aligned}
$$

The first term can be estimated from the estimate of $\phi$; for the second term we have

$$
\int_{1}^{\Lambda} \lambda^{-2 \sigma \beta-1} \phi(\lambda) d \lambda \leq C \int_{1}^{\Lambda} \lambda^{-\alpha \beta+n-\frac{n \beta}{p^{\prime}}-1} d \lambda=O\left(1+\Lambda^{n-\alpha \beta-\frac{n \beta}{p^{\prime}}}\right) .
$$


Putting the estimates together and expressing the estimate in terms of $p$ yields

$$
\int_{1}^{\Lambda} F(\lambda)^{\beta} \lambda^{n-1} d \lambda=O\left(1+\Lambda^{n-\alpha \beta-n \beta+\frac{n \beta}{p}}\right)
$$

and it follows that $F \in L^{\beta}\left((0, \infty), \lambda^{n-1} d \lambda\right)$ provided $n-\alpha \beta-n \beta+\frac{n \beta}{p}<0$. The proof is complete as the conditions on $\beta$ are equivalent to (3.4).

The role of $\sigma$ in this result simply specifies the possible range for the Lipschitz order $\alpha$ and the latter limits the range on $\beta$, in particular the lower bound. The following corollary gives the full higher dimensional generalization of $L^{1}$-integrability part of Theorem 1.2 .

Corollary 3.5. Let $1 \leq p \leq 2$ and suppose $\mu \in \mathcal{K}_{\sigma}$ for $2 \sigma>\frac{n}{p}$. If $f \in L^{p}\left(\mathbb{R}^{n}\right)$ and $\left\|M_{\mu}^{t} f-f\right\|_{p}=O\left(t^{\alpha}\right)$ for some $\frac{n}{p}<\alpha \leq 2 \sigma$, then $\widehat{f} \in L^{1}\left(\mathbb{R}^{n}\right)$ and Fourier inversion holds a.e.

The proof of this is simply to observe that under the stated conditions, the lower limit for $\beta$ in (3.4) is less than one. Hence $F \in L^{1}\left((0, \infty), \lambda^{n-1} d \lambda\right)$ and the result follows since $\|\widehat{f}(\lambda \cdot)\|_{L^{1}\left(S^{n-1}\right)} \leq \omega_{n-1}\|\widehat{f}(\lambda \cdot)\|_{L^{p}\left(S^{n-1}\right)}$.

Explicit realizations of this corollary would be with the measures defined in Propositions 2.8 and 2.10 for with $2 l>\frac{n}{p}$. As stated in Remark 2.13, examples of these measures and associated operators are connected with higher order smoothness. Hence the implication from the above corollary is that dimension dependent higher order smoothness conditions are needed to achieve a.e. Fourier inversion. This is in line with other works, e.g., Pinsky's work on Fourier inversion at a point [11, 12].

The following example indicates that the range for $\beta$ in Proposition 3.4 is best possible; this example is a based on one used by Titchmarsh [15] in one dimension.

Example 3.6. Let $\mu$ be a surface measure satisfying the hypothesis of Proposition 2.6, Let $1<p \leq 2$ and take $\frac{n}{p}-1<\gamma<\frac{n}{p}$. Consider the radial function

$$
f(x)=\frac{1}{|x|^{\gamma}+|x|^{n}} .
$$

Then $f \in L^{p}\left(\mathbb{R}^{n}\right)$. Claim: $\left\|M_{\mu}^{t} f-f\right\|_{p}=O\left(t^{\frac{n}{p}-\gamma}\right)$. Once the claim is proved, it follows that $F \in L^{\beta}\left((0, \infty), \lambda^{n-1} d \lambda\right)$ for $\frac{n}{n-\gamma}<\beta \leq p^{\prime}$. On the other hand, as $f$ is a radial function (and abusing notation), $F(\lambda)=\widehat{f}(\lambda)$ and since $f(x) \sim|x|^{-\gamma}$ for $x \rightarrow 0$, it follows that $F(\lambda) \sim \lambda^{\gamma-n}$ as $\lambda \rightarrow \infty$. Thus $F(\lambda)^{\frac{n}{n-\gamma}} \lambda^{n-1} \sim \lambda^{-1}$ and $F \notin L^{\frac{n}{n-\gamma}}\left((0, \infty), \lambda^{n-1} d \lambda\right)$.

In order to prove the claim, let $d$ be the diameter of the compact set $K$, let $t<1 / d$, and consider $x$ such that $|x|>d t$. Using the mean value theorem, $\left|M_{\mu}^{t} f(x)-f(x)\right| \leq t|\nabla f(x)|$. Write $f(x)=\tilde{f}(r), r=|x|$, then

$$
|\nabla f(x)|=\left|\tilde{f}^{\prime}(r)\right|=\frac{\gamma r^{\gamma-1}+n r^{n-1}}{\left(r^{\gamma}+r^{n}\right)^{2}} .
$$

It follows that

$$
\int_{|x|>d t}\left|M_{\mu}^{t} f(x)-f(x)\right|^{p} d x \leq \omega_{n-1} t^{p} \int_{d t}^{\infty}\left|\tilde{f}^{\prime}(r)\right|^{p} r^{n-1} d r=I_{1}(t)+I_{2},
$$


where $I_{1}(t)$ is the integral over $[d t, 1]$ and $I_{2}$ over the interval $[1, \infty)$. Then letting $C$ denote a generic constant, not necessarily the same in each occurrence, we have

$$
\begin{aligned}
I_{1}(t) & \leq C \int_{d t}^{1} r^{-p \gamma-p-n-1} d r=O\left(1+t^{n-p \gamma-p}\right) \\
I_{2} & \leq C \int_{1}^{\infty} r^{n-n p-p-1} d r=O(1),
\end{aligned}
$$

the latter because $n-n p-p<0$. Putting the pieces together yields the estimate

$$
\left(\int_{|x|>d t}\left|M_{\mu}^{t} f(x)-f(x)\right|^{p} d x\right)^{1 / p}=O\left(t^{p}+t^{\frac{n}{p}-\gamma}\right)=O\left(t^{\frac{n}{p}-\gamma}\right)
$$

the last estimate as $\frac{n}{p}-1<\gamma<\frac{n}{p}$. To finish the proof of the claim, consider the estimate

$$
\begin{array}{r}
\left(\int_{|x| \leq d t}\left|M_{\mu}^{t} f(x)-f(x)\right|^{p} d x\right)^{1 / p} \leq\left(\int_{|x| \leq d t}\left|M_{\mu}^{t} f(x)\right|^{p} d x\right)^{1 / p}+\left(\int_{|x| \leq d t}|f(x)|^{p} d x\right)^{1 / p} \\
=J_{1}(t)+J_{2}(t)
\end{array}
$$

Straightforward estimates show that $J_{2}(t)=O\left(t^{\frac{n}{p}-\gamma}\right)$ as $t \rightarrow 0$. To estimate the first piece we use Minkowski's inequality to write:

$$
J_{2}(t) \leq \int_{S}\left(\int_{|x| \leq d t}|f(x-t y)|^{p} d x\right)^{1 / p} d \mu(y)=\int_{S}\left(\int_{|z-t y| \leq d t}|f(z)|^{p} d z\right)^{1 / p} d \mu(y) .
$$

Now $\{z:|z-t y| \leq d t\} \subset\{z:|z| \leq 2 d t\}$ and hence

$$
J_{2}(t) \leq \int_{S}\left(\int_{|z| \leq 2 d t}|f(z)|^{p} d z\right)^{1 / p} d \mu(y)=O\left(t^{\frac{n}{p}-\gamma}\right) .
$$

This completes the proof of the claim.

\section{Further Results}

In this section two variations on the results presented above for the Euclidean Fourier transform are given. The first lies outside the realm of multipliers used above and concerns a variant for weak solutions of the Cauchy problem for the wave equation. Secondly, transference results are used to push the results of section 2 into the realm of the $n$-dimensional torus.

4.1. The Cauchy problem for the wave equation. Let $f \in L^{2}\left(\mathbb{R}^{n}\right)$ and let $u(x, t)$ be the weak solution of the Cauchy problem

$$
\begin{aligned}
& \text { PDE: } u_{t t}=\Delta u, x \in \mathbb{R}^{n}, t>0 \\
& \text { IC: }\left\{\begin{array}{c}
u(x, 0)=0 \\
u_{t}(x, 0)=f(x)
\end{array}, x \in \mathbb{R}^{n}\right.
\end{aligned}
$$

On the Fourier transform side, $\widehat{u}(\xi, t)=\frac{\sin t|\xi|}{|\xi|} \widehat{f}(\xi)$. Since $\mathcal{W}(t|\xi|)=\frac{\sin t|\xi|}{t|\xi|}$ is a bounded function, it forms a bounded multiplier operator on $L^{2}\left(\mathbb{R}^{n}\right)$. Moreover, it is easily shown 
that

$$
\left\|\frac{u(\cdot, t)}{t}-f(\cdot)\right\|_{2} \rightarrow 0, t \rightarrow 0 .
$$

Note that $\mathcal{W}(r)=j_{1 / 2}(r)$, and hence $|1-\mathcal{W}(r)| \asymp \min \left\{1, r^{2}\right\}$. Using the techniques from section 2 leads to the following proposition.

Proposition 4.1. Let $f \in L^{2}\left(\mathbb{R}^{n}\right)$ and let $u(x, t)$ be the weak solution of the Cauchy problem above. Then

and moreover

$$
\left[\int_{\mathbb{R}^{n}} \min \left\{1,(t|\xi|)^{4}\right\}|\widehat{f}(\xi)|^{2} d \xi\right]^{1 / 2} \asymp\left\|\frac{u(\cdot, t)}{t}-f(\cdot)\right\|_{2},
$$

$$
\left\|\frac{u(\cdot, t)}{t}-f(\cdot)\right\|_{2} \asymp\left\|M^{t} f(\cdot)-f(\cdot)\right\|_{2} .
$$

The second conclusion follows from the first and the $L^{2}$-result in Theorem [1.3, see also Example 2.7.

Remark 4.2. In the case $n=3, \frac{u(x, t)}{t}=M^{t} f(x)$, the spherical mean operator. This suggests that the proposition should have an generalization to other $L^{p}$-spaces. The difficulty is that the function $\mathcal{W}(t|\xi|)$ is not a bounded multiplier on all $L^{p}\left(\mathbb{R}^{n}\right)$. Rather, by embedding the spherical mean operator into an analytic family of operators, Stein [14] showed for $n \geq 4$ and $\frac{2 n}{n+1}<p \leq 2, \mathcal{W}(t|\xi|)$ is a bounded multiplier on $L^{p}\left(\mathbb{R}^{n}\right)$ and moreover

$$
\left\|\frac{u(\cdot, t)}{t}-f(\cdot)\right\|_{p} \rightarrow 0, t \rightarrow 0 .
$$

Under these conditions we then obtain the following inequality

$$
\left[\int_{\mathbb{R}^{n}} \min \left\{1,(t|\xi|)^{2 p^{\prime}}\right\}|\widehat{f}(\xi)|^{p^{\prime}} d \xi\right]^{1 / p^{\prime}} \leq c_{p}\left\|\frac{u(\cdot, t)}{t}-f(\cdot)\right\|_{p},
$$

for some positive constant $c_{p}$. This result is also valid for $n=1,2,3$ without restriction on $p$, i.e.. for $1 \leq p \leq 2$.

4.2. Transference to the torus. In this section the use of the hat notation $\widehat{f}$ will be used to denote Fourier coefficients as well as Fourier transforms; the meaning should be clear from context. Let $\mathbb{T}$ be the unit circle in the plane, $f \in L^{p}(\mathbb{T})$, and consider the following modulus of continuity:

$$
\omega_{p}[f](t)=\sup _{0<h<t}\|f(\cdot+h)+f(\cdot-h)-2 f(\cdot)\|_{L^{p}(\mathbb{T})} \cdot
$$

The analog of Theorem 1.1 in the context of Fourier series takes the following form.

Theorem 4.3. Let $1 \leq p \leq 2$. Then there is a constant $c_{p}>0$ such that for all $f \in L^{p}(\mathbb{T})$ :

- when $p=1$,

$$
\sup _{k \in \mathbb{Z}}\left[\min \left\{1,(t|k|)^{2}\right\}|\widehat{f}(k)|\right] \leq c_{1} \omega_{1}[f](t) ;
$$

- when $1<p<2$,

$$
\left[\sum_{k \in \mathbb{Z}} \min \left\{1,(t|k|)^{2 p^{\prime}}\right\}|\widehat{f}(k)|^{p^{\prime}}\right]^{1 / p^{\prime}} \leq c_{p} \omega_{p}[f](t)
$$


- when $p=2$,

$$
\left[\sum_{k \in \mathbb{Z}} \min \left\{1,(t|k|)^{4}\right\}|\widehat{f}(k)|^{2}\right]^{1 / 2} \asymp \omega_{2}[f](t) .
$$

The proof of this follows the ideas/methods given in section 2. Analogous to Proposition 1.2, the following corollary can be proved adapting the ideas of section 3 .

Corollary 4.4. Let $1 \leq p \leq 2$ and $f \in L^{p}(\mathbb{T})$ such that for some $0<\alpha \leq 2$,

$$
\|f(\cdot+t)+f(\cdot-t)-2 f(\cdot)\|_{L^{p}(\mathbb{T})}=O\left(t^{\alpha}\right) .
$$

Then $\sum_{k}|\widehat{f}(k)|^{\beta}$ converges for $\frac{p}{p+\alpha p-1}<\beta \leq p^{\prime}$. Moreover, if the above Lipschitz condition holds for some $\alpha>1 / p$, then the Fourier series of $f$ converges absolutely and uniformly on $\mathbb{T}$.

Looking toward extensions to higher dimension, let $\mathbb{T}^{n}=\mathbb{R}^{n} / \mathbb{Z}^{n}$ be the torus in $n$-dimensions. The measures $\mu \in \mathcal{K}_{\sigma}\left(\mathbb{R}^{n}\right)$ define Fourier multipliers on $L^{p}\left(\mathbb{R}^{n}\right)$ and can be transferred to Fourier multipliers on $L^{p}\left(\mathbb{T}^{n}\right)$ under mild regularity conditions on $\widehat{\mu}$ (see [10, Chapter 3]). Specifically, if $\widehat{\mu}$ is continuous on $\mathbb{R}^{n}$ (all of the examples given in this paper satisfy this condition), then the series,

$$
\sum_{k \in \mathbb{Z}^{n}} \widehat{\mu}(k) e^{i k \cdot x}
$$

is convergent to a measure, also denoted $\mu$ on $\mathbb{T}^{n}$. This class of transferred measures/multipliers is denoted $\mathcal{K}_{\sigma}\left(\mathbb{T}^{n}\right)$. Further, $\widehat{\mu}(t k), t>0$ and $k \in \mathbb{Z}^{n}$, defines a Fourier multiplier operator on $L^{p}\left(\mathbb{T}^{n}\right)$. The corresponding operator, also denoted $M_{\mu}^{t}$ is given by

$$
M_{\mu}^{t} f(x)=\sum_{k \in \mathbb{Z}^{n}} \widehat{\mu}(t k) \widehat{f}(k) e^{i k \cdot x}, f \in L^{p}\left(\mathbb{T}^{n}\right) .
$$

Here $\{\widehat{f}(k)\}_{k \in \mathbb{Z}^{n}}$ is the sequence of Fourier coefficients of $f$ and the series is convergent in $L^{p}$-norm. Given this set of ideas, the following variation of Theorem 2.2 is apparent.

Proposition 4.5. Let $n \geq 2, \mu \in \mathcal{K}_{\sigma}\left(\mathbb{T}^{n}\right)$, and let $1 \leq p \leq 2$. Then there is a constant $c_{p}>0$ such that for all $f \in L^{p}\left(\mathbb{T}^{n}\right)$ :

- when $p=1$,

$$
\sup _{k \in \mathbb{Z}^{n}}\left[\min \left\{1,(t|k|)^{2 \sigma}\right\}|\widehat{f}(k)|\right] \leq c_{1}\left\|M_{\mu}^{t} f-f\right\|_{1} ;
$$

- when $1<p<2$,

$$
\left[\sum_{k \in \mathbb{Z}^{n}} \min \left\{1,(t|k|)^{2 \sigma p^{\prime}}|\widehat{f}(k)|^{p^{\prime}}\right]^{1 / p^{\prime}} \leq c_{p}\left\|M_{\mu}^{t} f-f\right\|_{p} ;\right.
$$

- when $p=2$,

$$
\left[\sum_{k \in \mathbb{Z}^{n}} \min \left\{1,(t|k|)^{4 \sigma}|\widehat{f}(k)|^{2}\right]^{1 / 2} \asymp\left\|M_{\mu}^{t} f-f\right\|_{2} .\right.
$$


A version of this result may also be made in the vein of Theorem 2.12 using Pick's inequality on $\mathbb{T}^{n}$; this generalizes a result in $[9$ on the torus.

For $k \in \mathbb{Z}^{n}$, let $\|k\|=\max _{1 \leq j \leq n} k_{j}$ be the maximum norm. Due to the equivalence of norms on finite dimensional spaces, all of the estimates in the proposition above can be rewritten in terms of this norm. This fact will be useful in the proof of the following corollary generalizing Corollary 4.4 and in the vein of Proposition 3.4.

Corollary 4.6. Let $n \geq 2$, let $\mu \in \mathcal{K}_{\sigma}\left(\mathbb{T}^{n}\right)$ for some $\sigma>0$, and let $1 \leq p \leq 2$. If $f \in L^{p}\left(\mathbb{T}^{n}\right)$ such that for some $0<\alpha \leq 2 \sigma$,

$$
\left\|M_{\sigma}^{t} f-f\right\|_{L^{p}\left(\mathbb{T}^{n}\right)}=O\left(t^{\alpha}\right),
$$

then $\sum_{k}|\widehat{f}(k)|^{\beta}$ converges for $\frac{n p}{n p+\alpha p-n}<\beta \leq p^{\prime}$. Moreover, if $2 \sigma>\frac{n}{p}$ and the Lipschitz condition holds for some $\alpha>\frac{n}{p}$, then $\sum_{k \in \mathbb{Z}^{n}}|\widehat{f}(k)|$ converges, the Fourier series of $f$ converges absolutely and uniformly.

Proof. The proof is similar to that of Proposition 3.4 as follows. We consider the case $1<p \leq 2$, and take $N \in \mathbb{N}$. Then the estimate in the proposition above implies

$$
\sum_{\|k\| \leq N}\|k\|^{2 \sigma p^{\prime}}|\widehat{f}(k)|^{p^{\prime}} \leq C N^{(2 \sigma-\alpha) p^{\prime}}
$$

for some constant $C>0$. For $0<\beta<p^{\prime}$, this implies via Hōlder's inequality

$$
\sum_{\|k\| \leq N}\|k\|^{2 \sigma \beta}|\widehat{f}(k)|^{\beta}=O\left(N^{2 \sigma \beta+n-\alpha \beta-\frac{n \beta}{p^{\prime}}}\right) .
$$

Let

$$
\phi(N)=\sum_{1 \leq\|k\| \leq N}\|k\|^{2 \sigma \beta}|\widehat{f}(k)|^{\beta}=\sum_{l=1}^{N} l^{2 \sigma \beta} \sum_{\|k\|=l}|\widehat{f}(k)|^{\beta},
$$

and $\phi(0)=0$, then applying summation by parts

$$
\begin{aligned}
\sum_{1 \leq\|k\| \leq N}|\widehat{f}(k)|^{\beta} & =\sum_{l=1}^{N} l^{-2 \sigma \beta}(\phi(l)-\phi(l-1)) \\
& =\sum_{l=1}^{N-1}\left(l^{-2 \sigma \beta}-(l+1)^{-2 \sigma \beta}\right) \phi(l)+N^{-2 \sigma \beta} \phi(N) \\
& =I_{1}(N)+I_{2}(N) .
\end{aligned}
$$

The second piece is easily estimated using the estimate on $\phi$ to obtain $I_{2}(N)=O\left(N^{n-\alpha \beta-\frac{n \beta}{p^{\prime}}}\right)$.

For the first piece, we use the estimate on $\phi$ and simple estimates:

$$
\begin{aligned}
I_{1}(N) & \leq C \sum_{l=1}^{N} l^{-2 \sigma \beta}\left(1-\left(1+\frac{1}{l}\right)^{-2 \sigma \beta}\right) l^{2 \sigma \beta+n-\alpha \beta-\frac{n \beta}{p^{\prime}}} \\
& \leq C \sum_{l=1}^{N} l^{n-\alpha \beta-\frac{n \beta}{p^{\prime}}-1} \\
& =O\left(1+N^{n-\alpha \beta-\frac{n \beta}{p^{\prime}}}\right)
\end{aligned}
$$


Putting the estimates together we have

$$
\sum_{1 \leq\|k\| \leq N}|\widehat{f}(k)|^{\beta}=O\left(1+N^{n-\alpha \beta-n \beta+\frac{n \beta}{p}}\right),
$$

and the result follows.

Applying transference to the measures from Propositions 2.8 and 2.10 for $2 l>\frac{n}{p}$ yields explicit realizations for the second conclusion of this result.

\section{REFERENCES}

[1] J.J. Benedetto and J.D. Lakey, The definition of the Fourier transform for weighted inequalities, Jour. Func. Anal. 120 (2), p403-439 (1994).

[2] W.O.Bray and M.A.Pinsky, Growth properties of Fourier transforms via moduli of continuity, Jour. Func. Anal., 255, p2265-2285 (2009).

[3] W.O. Bray and M.A. Pinsky, Growth properties of the Fourier transform, Filomat 26:4, University of Nis, http://www.pmf.ni.ac.rs/pmf/publikacije/filomat/filomat_pocetna.php, p755-760 (2012); originally posted at arXiv:0910.1115v1 (2009).

[4] F. Dai and Z. Ditzian, Combinations of multivariate averages, Jour. Approx. Theory 131, p268-283 (2004).

[5] Z. Ditzian, Smoothness of a function and the growth of its Fourier transform or its Fourier coefficients, Jour. Approx. Theory 162, p980-986 (2010).

[6] Z. Ditzian, Relating smoothness to expressions involving Fourier coefficients or to a Fourier transform, Jour. Approx. Theory 164, p1369-1389 (2012).

[7] Z. Ditzian and K.G. Ivanov, Strong converse inequalities, Jour. d'Analyse Math. 61, p61-111 (1991).

[8] D. Gioev, Moduli of continuity and average decay of Fourier transforms: Two sided estimates, Cont. Math. 458, Amer. Math. Soc. p377-392 (2008).

[9] D. Gorbachev and S. Tikhonov, Moduli of smoothness and growth properties of Fourier transforms: Two-sided estimates, Jour. Approx. Theory 164, p1283-1312 (2012).

[10] L. Grafakos, Classical Fourier Analysis, 2nd edition, Graduate Texts in Math 249, Springer-Verlag (2008).

[11] M.A. Pinsky, Pointwise Fourier inversion and related eigenfunction expansions, Comm. Pure Appl. Math. 47, p653-681 (1994).

[12] M.A. Pinsky and M.E. Taylor, Pointwise Fourier inversion: a wave equation approach, Jour. Fourier Anal. Appl. 3, No. 6, p647-703 (1997).

[13] S.S. Platonov, The Fourier transform of functions satisfying the Lipschitz condition on rank one symmetric spaces, Siberian Math. Jour. 45, no. 6, p1108-1118 (2005).

[14] E. Stein, Maximal functions: spherical means, Proc. natl. Acad. Sci. 73 (7), p2174-2175 (1976).

[15] E.C. Titchmarsh, Introduction to the Theory of Fourier Integrals, Oxford University Press (1937).

Department of Mathematics, Missouri State University, Springfield, MO 65837

E-mail address: wbray@missouristate.edu 